\title{
A Fluorometric Biochemical Gas Sensor (Biosniffer) for Acetaldehyde Vapor Based on Catalytic Reaction of Aldehyde Dehydrogenase
}

\author{
Yuki Suzuki ${ }^{1}$, Ming Ye ${ }^{1}$, Kumiko Miyajima ${ }^{1,2}$, \\ Takahiro Arakawa'2, Shin-ichi Sawada ${ }^{3}$, Hiroyuki Kudo ${ }^{1,2}$, \\ Kazunari Akiyoshi ${ }^{3}$ and Kohji Mitsubayashi ${ }^{1,2, *}$ \\ ${ }^{1}$ Graduate School of Medical and Dental Sciences, Tokyo Medical and Dental University, \\ 1-5-45 Yushima, Bunkyo-ku, Tokyo 113-8549, Japan \\ ${ }^{2}$ Department of Biomedical Devices and Instrumentation, \\ Institute of Biomaterials and Bioengineering, Tokyo Medical and Dental University, \\ 2-3-10 Kanda-Surugadai, Chiyoda-ku, Tokyo 101-0062, Japan \\ ${ }^{3}$ Department of Polymer Chemistry, Graduate School of Engineering, Kyoto University, \\ Kyoto-Daigaku-Katsura, Nishikyo-ku, Kyoto 615-8510, Japan
}

(Received May 27, 2015; accepted September 24, 2015)

Key words: fiber-optic, biosensor, acetaldehyde, aldehyde dehydrogenase, gas sensor

A fiber-optic biochemical gas sensor (biosniffer) for the continuous monitoring of acetaldehyde (AA) vapor was constructed and tested. The biosniffer was constructed using a flow cell with an aldehyde dehydrogenase (ALDH) membrane onto a fiber-optic reduced nicotinamide adenine dinucleotide (NADH) measurement system. AA vapor was measured as fluorescence of NADH produced by the enzymatic reaction of ALDH. A phosphate buffer that contained oxidized nicotinamide adenine dinucleotide $\left(\mathrm{NAD}^{+}\right)$ was circulated into the flow cell to rinse NADH and the excessive substrates from the optode. A $340 \mathrm{~nm}$ ultraviolet light-emitting diode (UV-LED) was employed as an excitation source. The fluorescence of NADH $(\lambda=491 \mathrm{~nm})$ was measured coaxially with a spectrometer. Characterization of the biosniffer was carried out using a standard AA measurement system. As a result, the typical response is between 0.1 to $5.0 \mathrm{ppm} \mathrm{AA}$, which included the 8 -h reference exposure level $(0.16 \mathrm{ppm})$. The biosniffer is expected to be useful in the measurement of environmental AA.

\section{Introduction}

Acetaldehyde (AA) is one of the hazardous chemicals in air pollution with a penetrating fruity odor. Owing to its toxicity to humans, the maximum permitted concentrations of gaseous AA are set at 25 and 50 ppm by the American Conference of

${ }^{*}$ Corresponding author: e-mail: m.bdi@tmd.ac.jp 
Governmental Industrial Hygienists (ACGIH) and the Japanese Society of Occupational Health (JSOH), respectively. ${ }^{(1,2)}$ AA widely occurs in nature such as in coffee and bread and is produced by plants as part of their normal metabolism. It is also produced in human metabolism from alcohol consumption. In addition, it is found in adhesives, building materials, and cigarette smoke, and produced during secondary oxidation formation by photochemical reaction. ${ }^{(3)}$ Acetaldehyde is known as an irritant of the eye and upper airways, especially the nasal cavity, ${ }^{(4)}$ and AA is suspected of causing sick-building syndrome (SBS). ${ }^{(5)}$ Its reference exposure levels (RELs) are set by the Office of Environmental Health Hazard Assessment at $0.26 \mathrm{ppb}$ for acute effect, $0.16 \mathrm{ppm}$ for 8 -h exposure, and $0.08 \mathrm{ppb}$ for chronic exposure. ${ }^{(6)}$ For this reason, monitoring of the AA levels in indoor air is strongly required. Typically, low AA concentrations can be determined by high-performance liquid chromatography (HPLC) $^{(7,8)}$ or gas chromatography..$^{(9,10)}$ Although chromatographic methods result in accurate determination, they still have disadvantages in terms of cost, measurement time, and the need for skilled operators. On the other hand, the enzyme-based biosensor is one of the promising approaches for AA determination. There are several enzymes that catalyze the oxidation of AA (e.g., aldehyde ferredoxin oxidoreductase). Particularly, nicotinamide adenine dinucleotide $\left(\mathrm{NAD}^{+}\right)$-dependent dehydrogenase is suitable to be used in biosensors. Aldehyde dehydrogenase (ALDH) catalyzes the oxidation of AA in the presence of $\mathrm{NAD}^{+}$as an electron acceptor. In this reaction, acetic acid and reduced nicotinamide adenine dinucleotide (NADH) are produced. The enzymatic reaction is given as follows [eq. (1)].

$$
\text { acetaldehyde }+\mathrm{NAD}^{+} \rightarrow \text { acetic acid }+\mathrm{NADH}
$$

Previously, many papers that report on $\left(\mathrm{NAD}^{+}\right)$-dependent amperometric biosensors had been published. ${ }^{(11-16)}$ Although these biosensors are superior in terms of sampling volume, simplified structure, and measurement cost, there are several disadvantages to be considered. Most of the electroactive biomolecules such as catecholamine, ascorbic acid, and uric acid are oxidized at a potential close to that of NADH oxidation. In addition, the direct oxidation of NADH at bare electrodes results in electrode surface contamination (fouling) owing to the adsorption of the oxidized products of NADH, which results in poor sensitivity.(17)

Optical measurement is also commonly used in NADH determination. It is known that the absorbance at $340 \mathrm{~nm}$ is increased by the conversion of $\mathrm{NAD}^{+}$to NADH. NADH also produces fluorescence at $490 \mathrm{~nm}$ by the absorption at 340 $\mathrm{nm}$. We previously reported a fiber-optic NADH sensor using a UV-LED excitation system $^{(18)}$ and a fiber-optic biochemical gas sensor (biosniffer) for the monitoring of gaseous formaldehyde ${ }^{(19)}$ based on a NADH fluorometric detection. The formaldehyde biosniffer utilizes formaldehyde dehydrogenase to recognize the molecular structure of formaldehyde and is thus highly specific to formaldehyde. On the basis of the formaldehyde biosniffer, a high-sensitivity biosniffer for gaseous AA was constructed using ALDH. The aim of this work is to develop an ALDH-based biosniffer for the determination of AA in indoor air. In this paper, we describe the design, construction and characteristics of the ALDH biosniffer. 


\section{Experimental Methods}

\subsection{Reagents}

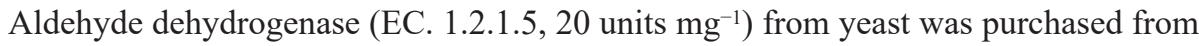
Boehringer Mannheim (France). $\mathrm{NAD}^{+}$was purchased from Oriental Yeast Co., Ltd. (Japan). Hydrophilic PTFE (H-PTFE, porosity: $80 \%$, pore size: $0.2 \mu \mathrm{m}$, JGWP14225) was received from Millipore Co., Ltd. (USA) as a carrier for enzyme immobilization. 2-methacryloyloxyethyl phosphorylcholine copolymerized with 2-ethylhexyl methacrylate (PMEH) was synthesized by the method shown in a previous report. ${ }^{(20)}$ Circulating buffer was prepared with $80 \mathrm{mmol} \mathrm{L}^{-1}$ phosphate buffer (PB) (pH 8.0), which contained $20 \mathrm{mmol} \mathrm{L}^{-1} \mathrm{NAD}^{+}$.

\subsection{Construction of the fiber-optic ALDH biosniffer}

The biosniffer consists of an excitation source, a photodetector, and an optode that converts the AA levels to the production of NADH. The excitation system utilizes a UV-LED (UVTOP ${ }^{\circledR}$ BL335, Sensor Electronic Technology, Inc., USA) using a customfabricated UV-LED power supply circuit (KLV Co., Ltd., Japan). A fiber-optic spectrometer (USB2000, Ocean Optics Inc., USA) was used as the photodetector. They were connected to each separated ends of a bifurcated optical fiber assembly (core size $600 \pm 10 \mu \mathrm{m}$, BIF600-UV/VIS, Ocean Optics Inc., USA) in this context. An optical fiber probe (F1000-900) was connected to the common end of the optical fiber assembly. To reduce the background level, a band-pass filter $(\lambda=340 \pm 10 \mathrm{~nm}$, BPF, Asahi Spectra Co., Ltd.) and a long-pass filter ( $\lambda>400 \mathrm{~nm}$, LPF, Asahi Spectra Co., Ltd.) were utilized in excitation and detection, respectively. The AA-sensitive optode was fabricated by attaching a flow cell with an ALDH-immobilized membrane to the end of the optical fiber probe. The flow cell has a cylindrical structure formed using a silicone tube $(\phi 2$ $\mathrm{mm})$ and a PMMA cell ( $\phi 4 \mathrm{~mm})$, forming a socket for the optical fiber probe. Microflow channels to supply the $\mathrm{PB}$, which contained $\mathrm{NAD}^{+}$, were formed on the boundary between the silicone and the PMMA as shown in Fig. 1. The ALDH-immobilized membrane was attached onto the end of the flow cell as a sensing diaphragm. ALDH (25 units $\left.\mathrm{cm}^{-2}\right)$ and PMEH solutions $\left(10 \%, 25 \mu \mathrm{cm}^{-2}\right)$ were mixed and spread carefully on the H-PTFE membrane filter. The membrane was cured in a refrigerator for $3 \mathrm{~h}$ at $4{ }^{\circ} \mathrm{C}$. The ALDH-immobilized membrane was cut out into $2 \times 2 \mathrm{~cm}^{2}$ and fixed onto the flow cell with a silicone O-ring. In this way, the biosniffer for AA vapor was constructed.

\subsection{Characterization of the fiber-optic ALDH biosniffer}

Figure 2 shows the experimental setup for the monitoring of AA vapor. In this system, the AA vapor was supplied from a standard gas generator (PD-1B-2, GASTEC corporation, Japan). The flow rate was controlled using a mass flow controller with a needle-bulb regulator (RK1200, Koflok, Japan). The PB that contained $\mathrm{NAD}^{+}$was circulated into a flow cell by an intelligent pump (FLOM Co., Ltd., Japan). Usually, the enzyme loses its specific activity if the chemical conditions are inadequate (e.g., dry condition, inappropriate temperature, $\mathrm{pH}$ ). The flow of $\mathrm{PB}$ kept the enzyme membrane wet, supplied $\mathrm{NAD}^{+}$, and also removed the reaction products (including $\mathrm{NADH}$ ) and 


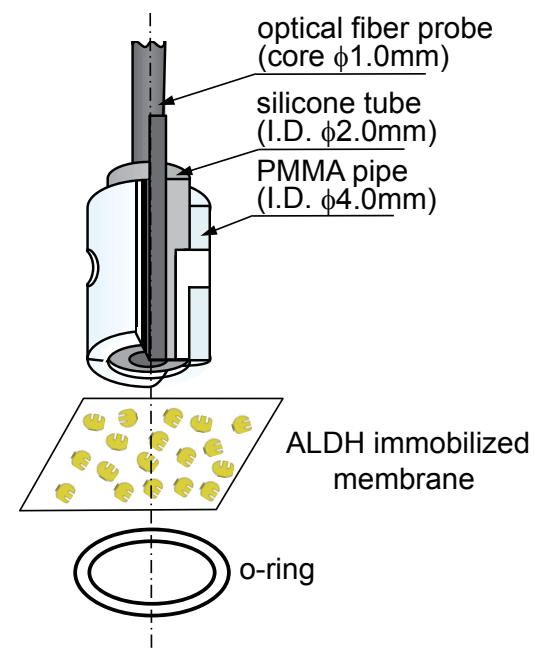

(a)

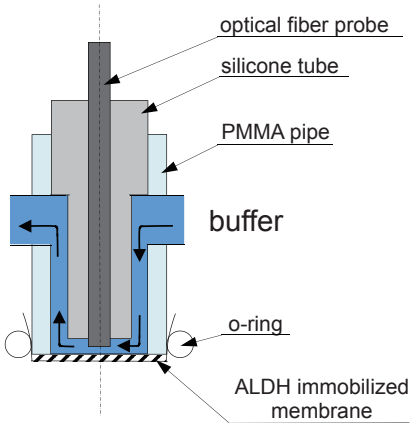

(b)

Fig. 1. (Color online) Structure of flow cell with ALDH-immobilized membrane (a) and cross section of the flow cell of the ALDH biosniffer (b).

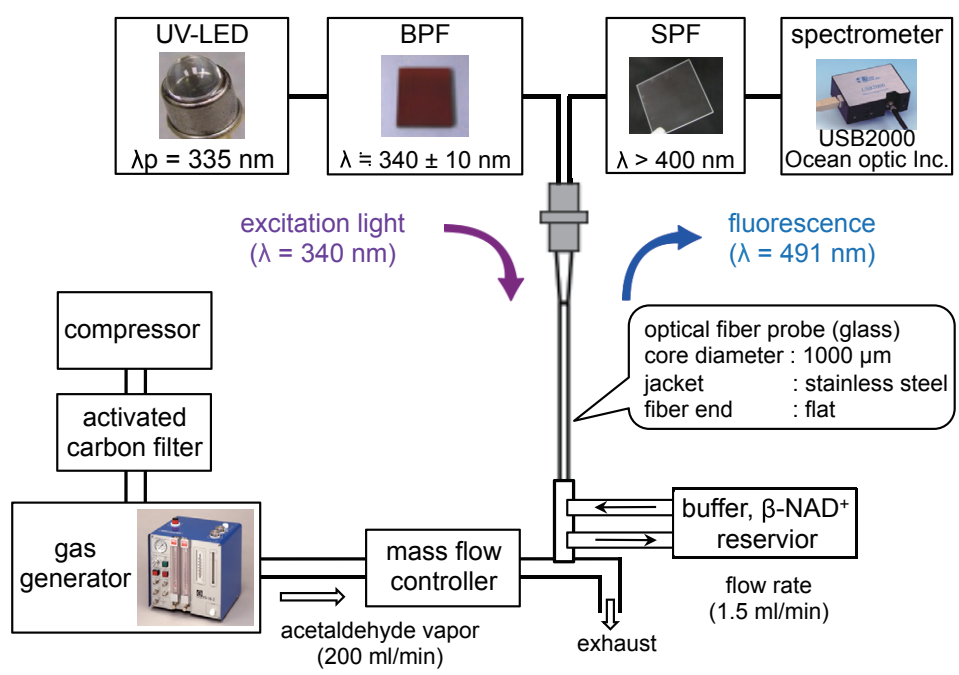

Fig. 2. (Color online) Experimental setup for characterization of the fiber-optic biosniffer. The biosniffer measures AA vapor as fluorescence of NADH, which is produced at the enzyme membrane. $\mathrm{PB}$ that contained $\mathrm{NAD}^{+}$was circulated with a flow rate of $1.5 \mathrm{ml} \mathrm{min}^{-1}$. The capacity of the reservoir is $500 \mathrm{~mL}$. 
excessive substrates effectively. Therefore, the reaction condition was always refreshed by the buffer circulation.

The AA biosniffer was characterized by supplying the standard gas to the sensing region via a Teflon tube. Clean air was flowed into the Teflon tube first, and then the AA vapor was applied to the biosniffer. The total flow rate was kept at $200 \mathrm{ml} \mathrm{min}^{-1}$. Clean air was also used as the carrier gas to adjust the AA levels. The fluorescences of the NADH were measured using a spectrometer. The experiment was repeated thrice.

\section{Results and Discussion}

\subsection{Behavior of the ALDH biosniffer}

The fluorescence response to AA vapor was first investigated using the standard gas measurement system. Figure 3 shows the typical responses of the AA biosniffer to various concentrations of gaseous AA. As shown in the figure, fluorescent spectra with the peak wavelength of $491 \mathrm{~nm}$, which is similar to that in a previous study, ${ }^{(21)}$ were confirmed. This indicates that NADH was produced as expected at the enzyme membrane by the catalytic reaction of ALDH. The intensity increased as the AA level increased. Hence, exposure to AA vapor induced the increase in the fluorescence intensity. From this result, the signal level was approximately $45 \%$ compared with that of the FALDH biosniffer. ${ }^{(19)}$ This can be associated with the difference in the catalytic activities of ALDH (with AA and formaldehyde).

\subsection{Characteristics of the ALDH biosniffer}

The calibration curve for AA is shown in Fig. 4. The analytical curve was described using the following equation [eq. (2)]:

$$
\text { Intensity }(\text { counts })=-1.12+27.90[\text { acetaldehyde }(\mathrm{ppm})]
$$

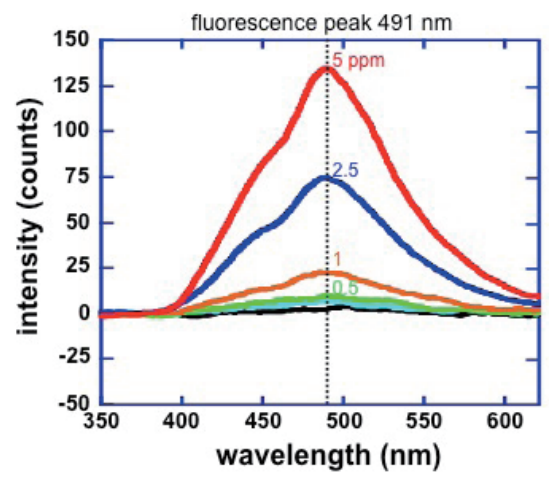

Fig. 3. (Color online) Fluorescent spectra of the fiber-optic biosniffer for various concentrations of AA vapor. 


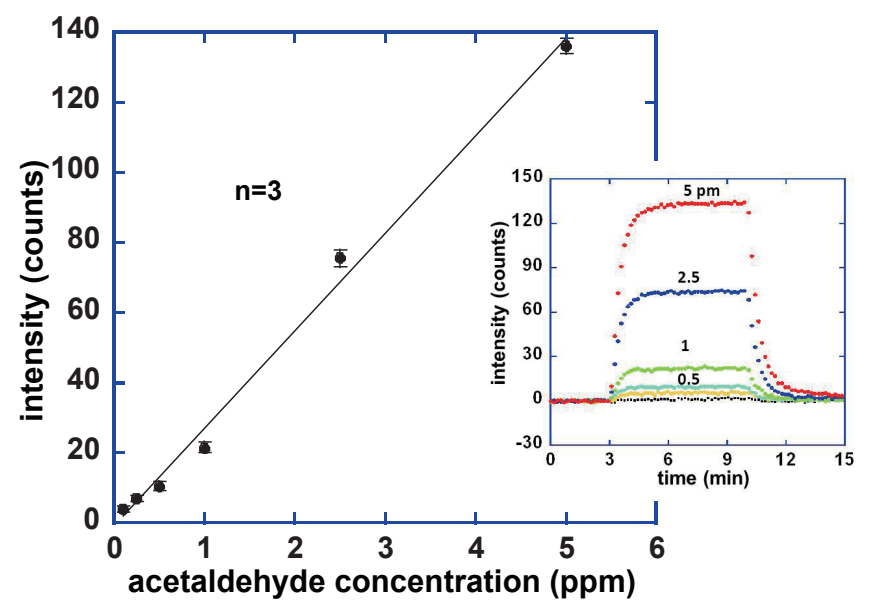

Fig. 4. (Color online) Calibration curve for AA vapor. The fluorescence intensity related to the AA vapor from 0.1 to $5 \mathrm{ppm}$. The inset shows the responses to AA vapor. The steady value is determined by the balance of NADH production at the flow cell and the rinsing efficiency of the $\mathrm{PB}$ circulation.

with a correlation coefficient of 0.988 . The calibration range was 0.1 to $5.0 \mathrm{ppm}$, which included the 8 -h REL $(0.16 \mathrm{ppm})$. From this result, the intensities had a direct relationship with the AA levels. Therefore, the biosniffer was expected to be useful in the measurement of environmental AA.

The inset of Fig. 4 shows the real-time response at $491 \mathrm{~nm}$ to various concentrations of gaseous AA. When the AA vapors were supplied to the sensing region, the fluorescence intensity increased and reached the steady state immediately. The output level decreased to the initial state when the AA vapor was disrupted. Those responses indicate an increase in the NADH level at the enzyme membrane owing to the ALDH reaction and the immediate removal of NADH from the sensing region by the buffer flow. The flow rate of $\mathrm{PB}$ was optimized to $1.5 \mathrm{ml} \mathrm{min}^{-1}$. In the case of a lower flow rate, it is not sufficient to remove the NADH. Conversely, at a high flow rate, the recovery time becomes faster while the fluorescence intensity decreases. This supports the fact that the biosniffer measures the gas level as a balance of the production and removal rates of NADH. The fast response to AA vapor provides an advantage in onsite measurement compared with conventional chromatographic methods such as HPLC or GC and other biochemical determinations such as colorimetric methods. The time to reach $95 \%$ of the steady state was $102 \mathrm{~s}$, which can compete with solid-state gas sensors for monitoring purposes. ${ }^{(7,9)}$ Thus, the sensor confirmed that it is applicable to the realtime monitoring of AA.

Selectivity to possible interferences was also investigated. Since ALDH is also active with formaldehyde, the ALDH biosniffer also responds to formaldehyde. However, it 
can be compensated using the FALDH biosniffer because FALDH is not active with AA. In addition, the ALDH biosniffer was not active with methanol (2.0 ppm), ethanol (2.0 $\mathrm{ppm})$, benzene $(2.0 \mathrm{ppm})$, and acetone $(2.0 \mathrm{ppm}){ }^{(22)}$

Further improvement in the sensitivity can be expected by modifying the optical system. As reported in a previous study, ${ }^{(19)}$ the lower detection limit can be improved (typically 10 times) by the use of a photomultiplier tube (PMT) as the photodetector. Acetaldehyde is produced in natural processes including alcohol metabolism as mentioned earlier. With such a system, the contactless and nondestructive assessment of food quality or medical applications (e.g., by AA in breath or transdermal gases) will also be expected.

\section{Conclusions}

A continuous, high-sensitivity and high-selectivity fiber-optic biochemical gas sensor based on the catalytic reaction of ALDH was developed. The sensing system used a UV-LED as an excitation system for the purpose of miniaturization and ease of use. By utilizing the cylindrical flow cell with the enzyme-immobilized membrane, the realtime monitoring of gaseous AA was successfully performed. The fluorescence signal was proportional to the AA level from 0.1 to $5.0 \mathrm{ppm}$, which included the 8 -h REL $(0.16 \mathrm{ppm})$. Although aldehyde dehydrogenase is active with formaldehyde, this can be compensated with the combined use of FALDH or it can also be improved by using the enzyme, which is highly specific for AA. The response of the biosniffer to AA vapor was sufficiently fast for application to environmental monitoring. Medical or food analysis applications are also expected with the further improvement in sensitivity, because of its high selectivity to alcohol vapor.

\section{Acknowledgements}

This work was partly supported by the Japan Society for the Promotion of Science (JSPS) Grants-in-Aid for Scientific Research System, Japan Science and Technology Agency (JST), and Ministry of Education, Culture, Sports, Science and Technology (MEXT) Special Funds for Education and Research "Research Promotion of Neo-Biology".

\section{References}

1 ACGIH: Documentation of the Threshold Limit Values and Biological Exposure Indices, 6th ed. (ACGIH Publishing, Cincinnati, OH, 1991).

2 Japanese Society of Occupational Health (JSOH): J. Occup. Health 53 (2011) 395.

3 IARC: International Agency for Research on Cancer (IARC Publishing, Lyons, France, 1999) p. 319.

4 IPCS: Homepage of IPCS, http://www.inchem.org/documents/ehc/ehc/ehc167.htm (accessed May 2015).

5 T. Takigawa, Y. Saijo, K. Morimoto, K. Nakayama, E. Shibata, M. Tanaka, T. Yoshimura, H. Chikara and R. Kishi: Sci. Total Environ. 417-418 (2012) 61.

6 OEHHA: Homepage of OEHHA, http://oehha.ca.gov/air/allrels.html (accessed May 2015). 
7 M. S. Gandelman and J. W. Birks: J. Chromatogr. 242 (1982) 21.

8 F. Lipari and S. J. Swarin: J. Chromatogr. 247 (1982) 297.

9 A. Yasuhara and T. Shibamoto: J. Chromatogr. A 672 (1994) 261.

10 Y. Mori, K. Tsuji, S. Setsuda, S. Goto, S. Onodera and H. Matsushita: Jpn. J. Toxicol. Environ. Health 42 (1996) 500.

11 S. Yamazaki, K. Miki, K. Kano and T. Ikeda: J. Electroanal. Chem. 516 (2001) 59.

12 M. E. Ghica, R. Pauliukaite, N. Marchand, E. Devic and C. M. Brett: Anal. Chim. Acta 591 (2007) 80.

13 T. Noguer and J. L. Marty: Enzyme Microb. Technol. 17 (1995) 453.

14 W. B. Nowall and W. G. Kuhr: Anal. Chem. 67 (1995) 3583.

15 M. Musameh, J. Wang, A. Merkoci and Y. H. Lin: Electrochem. Commun. 4 (2002) 743.

16 L. Gorton: J. Chem. Soc., Faraday Trans. 182 (1986) 1245.

17 S. A. Kumar and S. M. Chen: Sensors Basel 8 (2008) 739.

18 H. Kudo, M. Sawai, X. Wang, T. Gessei, T. Koshida, K. Miyajima H. Saito and K. Mitsubayashi: Sens. Actuators, B 141 (2009) 20.

19 H. Kudo, X. Wang, Y. Suzuki, M. Ye, T. Yamashita, T. Gessei, K. Miyajima, T. Arakawa and K. Mitsubayashi: Sens. Actuators, B 161 (2012) 486.

20 H. Kudo, T. Yagi, M. X. Chu, H. Saito, N. Morimoto, Y. Iwasaki, K. Akiyoshi and K. Mitsubayashi: Anal. Bioanal. Chem. 391 (2008) 1269.

21 H. Kudo, M. Sawai, Y. Suzuki, X. Wang, T. Gessei, D. Takahashi, T. Arakawa and K. Mitsubayashi: Sens. Actuators, B 147 (2010) 676.

22 K. Mitsubayashi, H. Matsunaga, G. Nishio, S. Toda, Y. Nakanishi, H. Saito, M. Ogawa and K. Otsuka: Sens. Actuators, B 108 (2005) 660. 